\title{
Espectro de hallazgos por TC multidetector en el trauma esplénico y hepático
}

\section{Multidetector CT Findings in Splenic and Liver Trauma}

\author{
Andrés C. Ghezzo ${ }^{1}$ Daniel O. Adri ${ }^{1} \quad$ Ernestina Gentile $^{1} \quad$ Ezequiel Levy Yetati $^{1}$ \\ ${ }^{1}$ Servicio de Diagnóstico por Imágenes, Hospital Italiano, Ciudad \\ Address for correspondence Daniel O. Adri, Servicio de Diagnóstico \\ Autónoma de Buenos Aires, Argentina \\ por Imágenes, Hospital Italiano, Ciudad Autónoma de Buenos Aires, \\ Rev Argent Radiol 2018;82:168-174. \\ Argentina (e-mail: danieladri33@gmail.com).
}

\section{Resumen \\ Palabras Clave \\ - trauma abdominal \\ - tomografía computada \\ - lesión esplénica hepática \\ - ruptura esplénica}

El trauma abdominal cerrado puede ser causa de lesiones en varios órganos sólidos, siendo el bazo y el hígado más frecuentemente afectados en ese tipo de situaciones. La tomografía computada multicorte (TCMC), es el método de imágenes de elección en la evaluación de pacientes que sufrieron un trauma cerrado abdominal gracias a que aporta un rápido y preciso diagnóstico y permite un eficaz tratamiento. El objetivo de este trabajo, es describir el espectro de las lesiones esplénicas y hepáticas encontradas en el trauma abdominal cerrado.

Blunt Abdominal Trauma can cause lesions in several abdominal solid organs. The spleen and liver are most frequently injured in such situations. Multi Slice Computed Tomography (MSCT) is the method of choice in the evaluation of patients who suffered a closed abdominal trauma to provide a quick and accurate diagnosis and allow faster and more effective treatment. This pictorial essay aims to describe the most frequent spleen and liver injuries in blunt abdominal trauma.

- liver/injuries

- splenic rupture

\section{Introducción}

El trauma es la principal causa de muerte en hombres y mujeres de menos de 45 años en Estados Unidos y en nuestro país, siendo la cuarta causa en todas las edades. Los accidentes de motocicleta - automóvil, caída de alturas y accidentes deportivos son los motivos primarios. ${ }^{1,2}$

En el trauma abdominal cerrado, el bazo es el órgano más frecuentemente afectado, encontrándose lesión entre un $40 \%$ a un $45 \%$ de los casos. El hígado es el segundo con un $18 \%$ aproximadamente. $^{3,4}$ El trauma hepático aislado es raro, acompañándose de lesiones en otros órganos en un $77 \%$ a un $90 \% .{ }^{5}$ En el trauma esplénico, los hallazgos en el examen semiológico y de laboratorio iniciales suelen ser inespecíficos, ${ }^{6}$ mientras que en el hepático la elevación del nivel de las transaminasas en sangre es $100 \%$ sensible y $92,3 \%$ específico. ${ }^{7}$ Sin embargo, en ambos casos, el método de elección para su evaluación y descarte de lesión ante un traumatismo abdominal continúa siendo la TCMC, por su velocidad, accesibilidad y eficacia diagnóstica. ${ }^{8}$ Además, puede ayudar a detectar lesiones asociadas como, por ejemplo, daño en la pared abdominal, retro peritoneo y exclusión de lesiones en órganos netamente quirúrgicos como intestino y páncreas. ${ }^{9,10}$

A pesar de que la decisión de una conducta quirúrgica está basada en un criterio clínico, la TCMC ha influenciado la

\section{received}

December 14, 2017

accepted

April 29, 2018

published online

June 8, 2018
DOI https://doi.org/

10.1055/s-0038-1657774.

ISSN 1852-9992.
Copyright (c) 2019, Sociedad Argentina de Radiología. Publicado por Thieme Revinter Publicações Ltda., Rio de Janeiro, Brazil. Todos los derechos reservados.
License terms

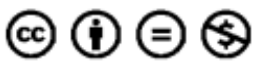


tendencia actual en el manejo de las lesiones esplénicas y hepáticas dando confianza a los cirujanos en adoptar una conducta no quirúrgica inicial en relación a los hallazgos, lo cual ha disminuido el número de laparotomías innecesarias. ${ }^{11-13}$ El objetivo de este trabajo, es describir el espectro de las lesiones esplénicas y hepáticas encontradas en el trauma abdominal cerrado.

\section{Discusión}

El trauma es una de las principales causas de morbi mortalidad y está en 4to lugar en EEUU, luego de enfermedades cardiovasculares, cáncer y enfermedades respiratorias. Tanto el bazo como el hígado son los dos órganos más afectados, seguido de las lesiones genitourinarias. ${ }^{14}$ En el trauma cerrado abdominal con afectación esplénica, los datos de laboratorio suelen ser inespecíficos pudiendo encontrarse sólo disminución del hematocrito. ${ }^{6}$ Por el contrario, en el traumatismo hepático, se puede encontrar alteración principalmente del hepatograma. La elevación del nivel de las transaminasas en sangre es $100 \%$ sensible y $92,3 \%$ específico. $^{7}$
La ecografía en protocolo Focused abdominal sonography in trauma (FAST), suele ser el primer método utilizado en el abdomen agudo en general, útil en la detección de líquido, principalmente la presencia o no de hemoperitoneo con una sensibilidad del 90 al 93\%. En pacientes clínicamente estables se debe realizar una TCMC cuando el Eco FAST es negativo para hemoperitoneo, ya que existe lesión intraabdominal en un estimado $29 \%$ de los casos y haber cambio de conducta terapéutica, debido a los hallazgos en TCMC, en un $16 \%{ }^{15}$

La TCMC continúa siendo el método de referencia en el trauma abdominal cerrado en pacientes hemodinámica-mente estables. ${ }^{16,17}$ Con un protocolo de cuatro fases, posee una sensibilidad del $95 \%$, una especificidad del $100 \%$, un valor predictivo positivo del $100 \%$ y un valor predictivo negativo del 78\%, según algunas series, en el diagnóstico de las diferentes lesiones de órganos sólidos. ${ }^{3}$ A su vez, es posible clasificar en grados las diferentes lesiones (sistemas de gradación de lesiones esplénicas y hepáticas basadas en TCMC desarrollado por The American Association for the Surgery of Trauma), dar una aceptable estimación de volumen de hemoperitoneo y detectar la presencia y localización de la hemorragia arterial activa, pseudoaneurismas y fístulas arterio-venosas.
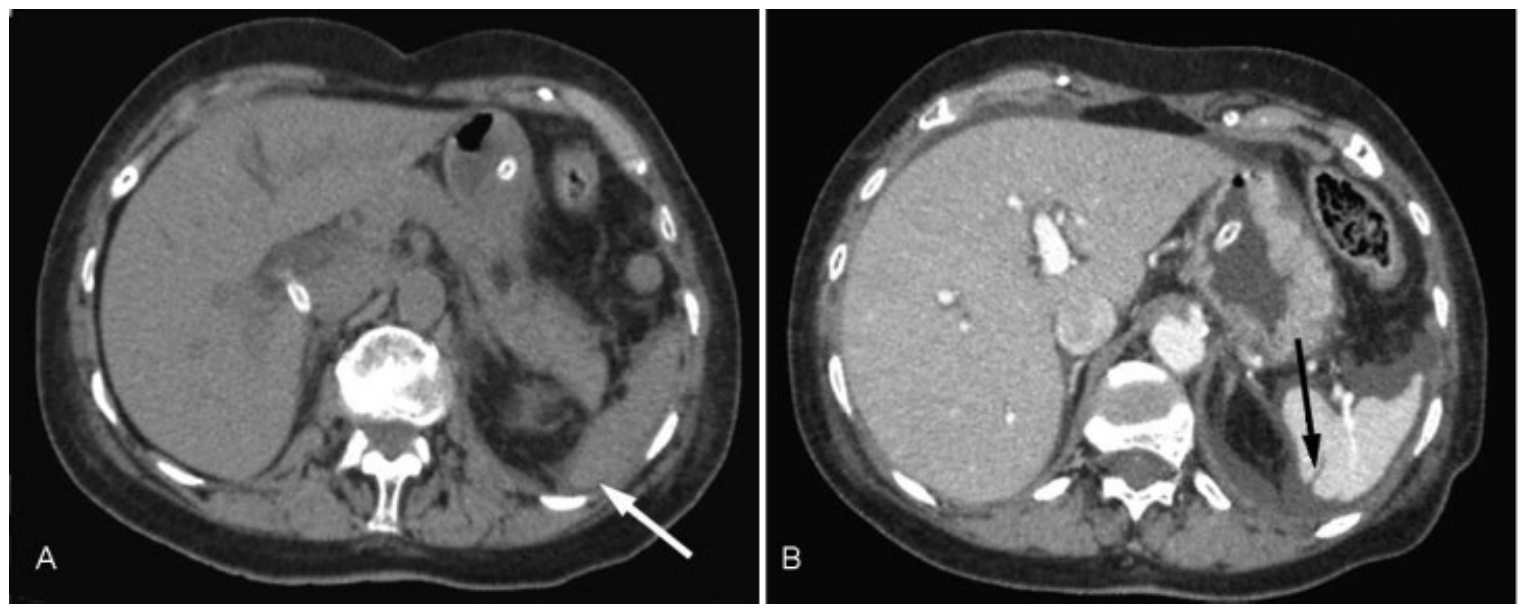

Fig. 1 Paciente de 84 años con politraumatismo grave de vehículo versus peatón, en (A) plano axial sin contraste intravenoso (IV) se evidenció banda hipodensa compatible con laceración focal lineal en polo inferior esplénico (flecha blanca), asociado a líquido libre periesplénico; en (B) nótese la ausencia de realce de la misma tras la administración del contraste IV. (Flecha negra).
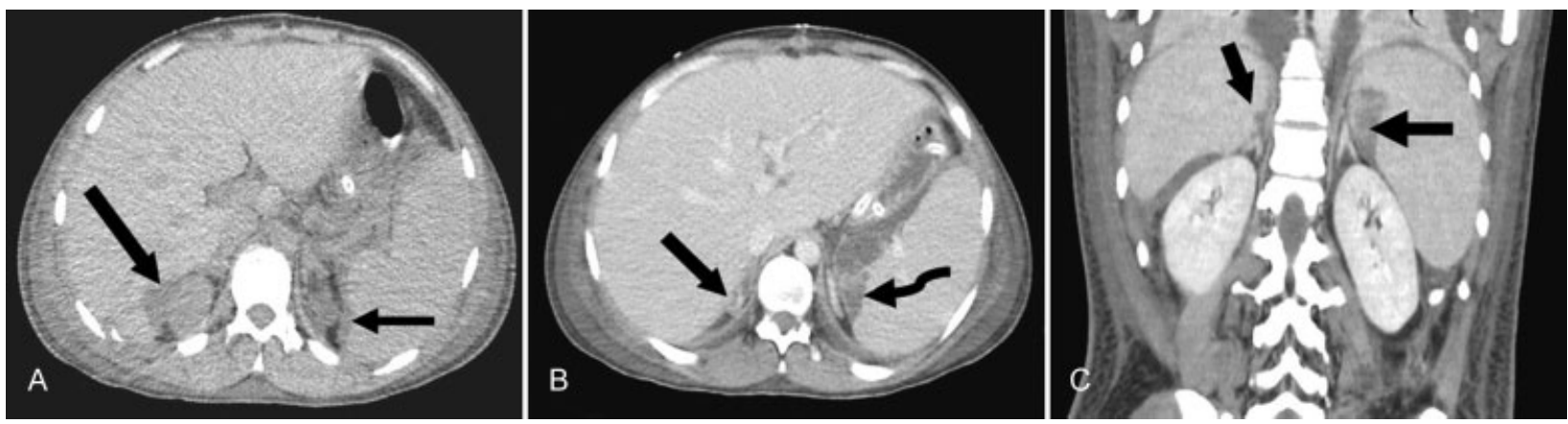

Fig. 2 Paciente de 26 años que presentó politraumatismo secundario a la colisión de su motocicleta contra un camión, en (A) plano axial sin contraste IV se evidenció hipodensidad focal de bordes irregulares en segmento VI hepático (flecha oblicua) e hipodensidad focal en polo superior esplénico (flecha horizontal); en (B) plano axial con contraste IV, fase portal: falta de realce de ambas lesiones: hepática (flecha oblicua) y esplénica (flecha horizontal) correspondiente a laceraciones. (C) Reconstrucción coronal en fase portal de laceración hepática (flecha oblicua) y esplénica (flecha horizontal). 

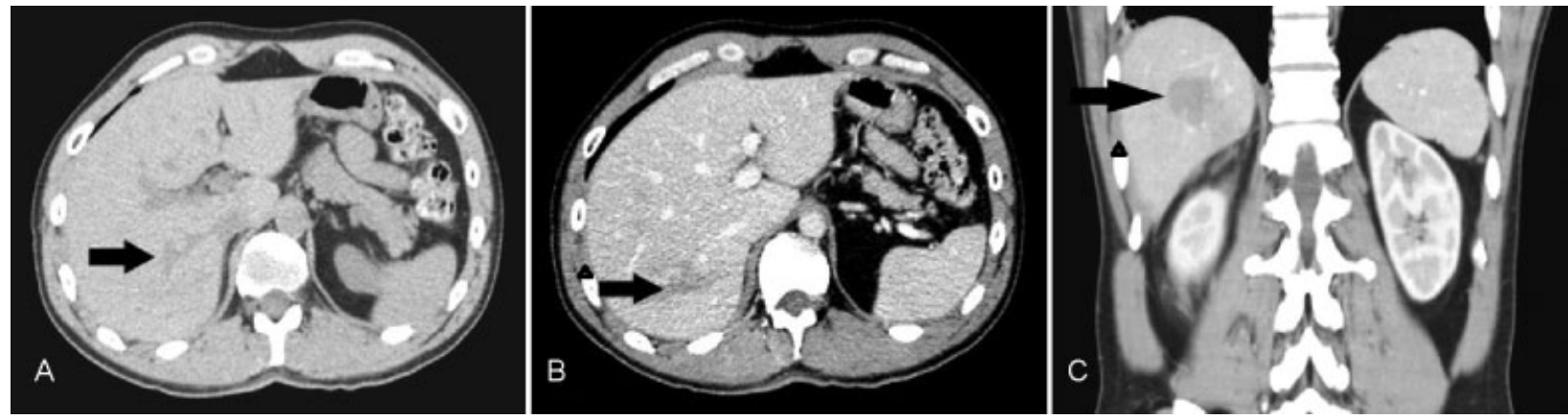

Fig. 3 Paciente de 19 años con politraumatismo por accidente en motocicleta, en (A) plano axial sin contraste: lesión focal hipodensa en segmentos VI-VII hepático (flecha). Tras la administración de contraste IV (B) se evidenció la misma lesión sin realce (flecha), asociado a escaso líquido peri hepático (cabeza de flecha). (C) Reconstrucción coronal de fase portal que demostró laceración hepática para su correcta localización espacial y extensión (flecha).
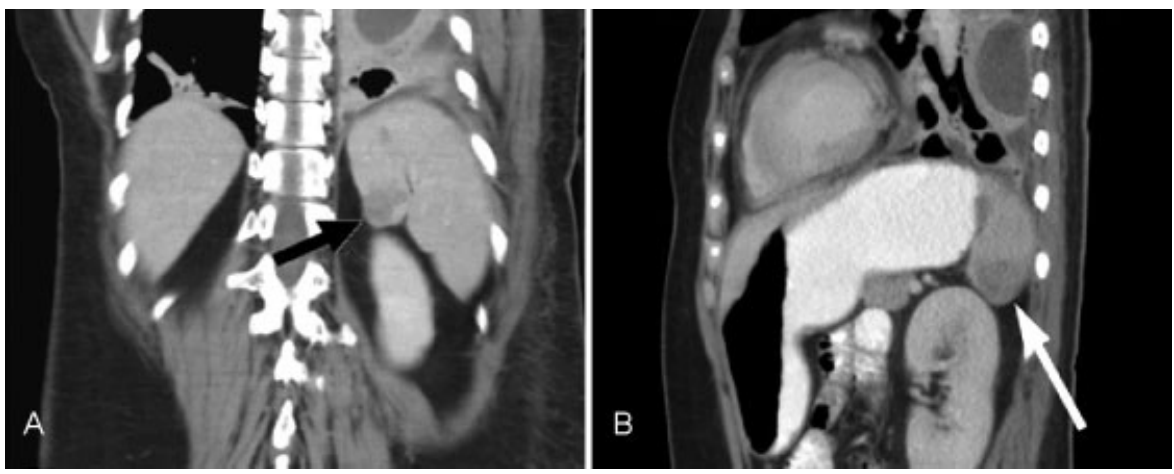

Fig. 4 Paciente de 60 años con antecedente de insuficiencia renal, presentó politraumatismo por caída de dos metros de altura. En (A) podemos observar una reconstrucción coronal con contraste IV que evidenció área focal esplénica bien definida de bordes irregulares heterogénea correspondiente a hematoma intra parenquimatoso (flecha). (B) Reconstrucción sagital de la misma para su correcta localización espacial y extensión (flecha).
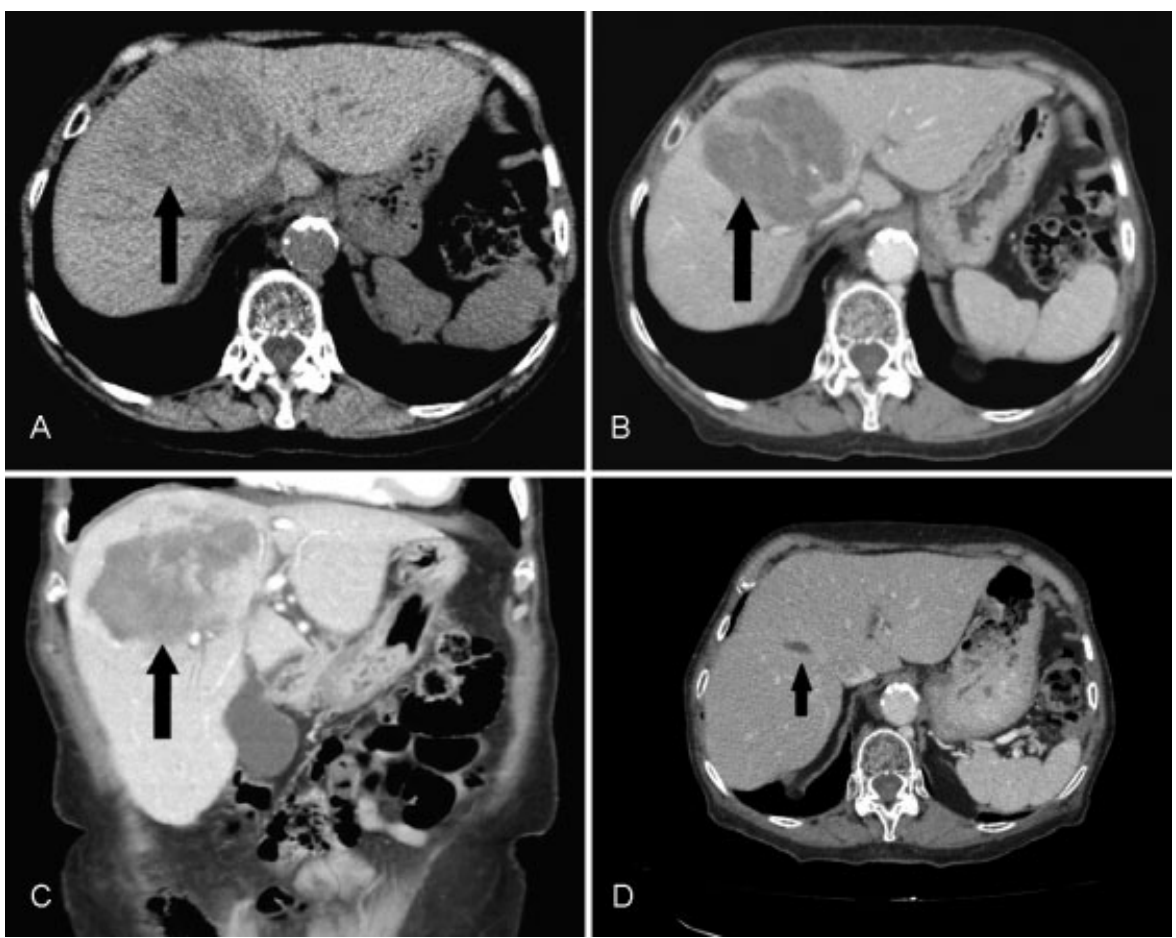

Fig. 5 Paciente de 84 años que presentó caída de propia altura con posterior manejo conservador satisfactorio. En (A) plano axial sin contraste IV: lesión hipodensa, con márgenes mal definidos, que compromete a los segmentos IV, Vy VIII hepáticos (flecha). En fase portal (B) se evidenció delimitación de lesión (flecha), y el parénquima normal subyacente de bordes ahora bien definidos. (C) Reconstrucción coronal en fase portal que muestra hematoma intra parenquimatoso que compromete a los segmentos IV, V y VIII hepáticos (flecha). (D) Control anual con resolución (flecha). 
Las limitaciones diagnósticas se basan en la baja sensibilidad en la detección de lesiones pancreáticas, vesicales, del mesenterio y hemorragia venosa, en sus estadios tempranos. ${ }^{18,19}$ A continuación, se mencionan los principales hallazgos patológicos mediante TCMC:

A) Laceración: Es un área de hipoperfusión del parénquima visualizada como una hipodensidad lineal o irregular que no realza tras la administración de contraste IV. El parénquima esplénico y hepático normal realza intensamente, lo cual permite diferenciar esas áreas con relativa facilidad.

En el bazo no deben ser confundidas con lobulaciones las cuales son variantes anatómicas normales. Cuando la lesión atraviesa la cápsula o el hilio se le suele denominar "fractura" esplénica (-Figs. 1 y 2). En el hígado, las laceraciones que se extienden por la fisura transversa hepática están comúnmente asociadas a la lesión de los conductos biliares, los cuales pueden producir bilomas (-Figs. 2 y 3 ).

B) Hematoma intraparenquimatoso: Es una colección de sangre dentro del parénquima, visualizada como área bien delimitada, hipodensa en estadios tempranos (sangre no coagulada) y espontáneamente hiperdensa (sangre coagulada) en la fase sin contraste IV. Sin realce tras la administración de contraste IV (-Figs. 4 y 5 ).

C) Hematoma subcapsular: Colección de sangre que produce una impronta sobre el parénquima subyacente de forma lenticular, con cápsula esplénica y hepática intacta de comportamiento tomográfico similar al hematoma intraparenquimatoso (-Figs. 6 ys 7).

D) Hemorragia activa: La extravasación activa suele verse como un área lineal o irregular de contraste en el parénquima esplénico, espacio subcapsular o intraperitoneal. Para diferenciarlo de un pseudoaneurisma se utiliza la fase tardía esperando la falta de lavado del contraste administrado en ese último. Se visualiza en la fase con contraste como un material isodenso a las arterias contrastadas. Puede verse dentro de las laceraciones material hiperdenso en la fase sin contraste (-Figs. 8 y 9 ).

E) Pseudoaneurismas: son definidos como un hematoma encapsulado comunicado con la luz de un vaso lesionado sin la totalidad de las capas, de baja prevalencia. Pueden visualizarse como un engrosamiento o nódulo espontáneamente hiperdenso con realce tras la administración de contraste IV en fase arterial, portal y tardía. En ocasiones, es posible seguir su origen en un vaso
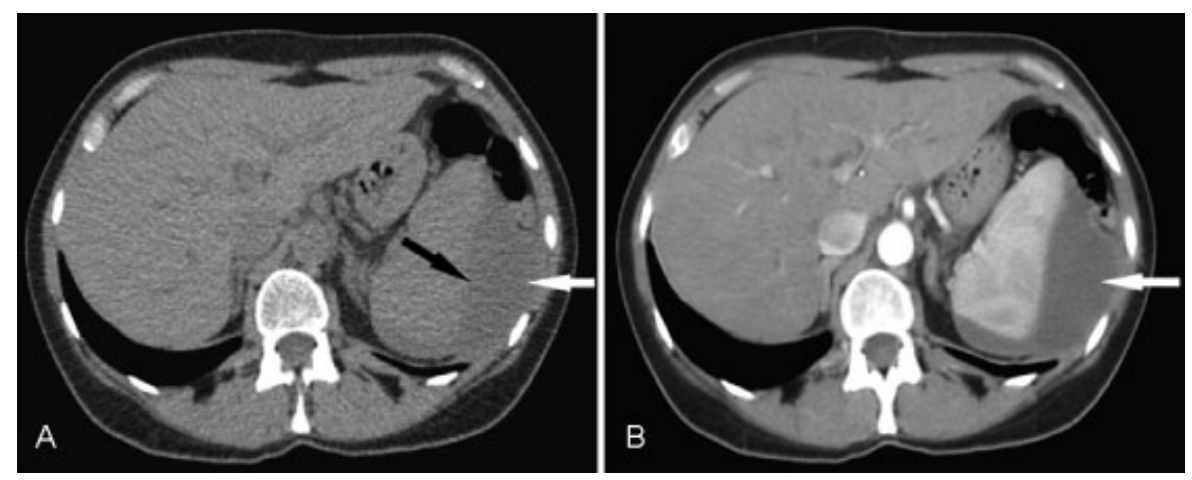

Fig. 6 Paciente de 50 años presentó trauma contuso contra pared de su casa al tropezar. En (A) podemos observar un plano axial sin contraste IV: colección periesplénica de bordes bien definidos, que produjo impronta sobre el parénquima esplénico subyacente (entre flechas negra y blanca) compatible con hematoma subcapsular esplénico. (B) Plano axial en fase arterial que demostró colección subcapsular sin realce, con cápsula esplénica indemne (flecha blanca).
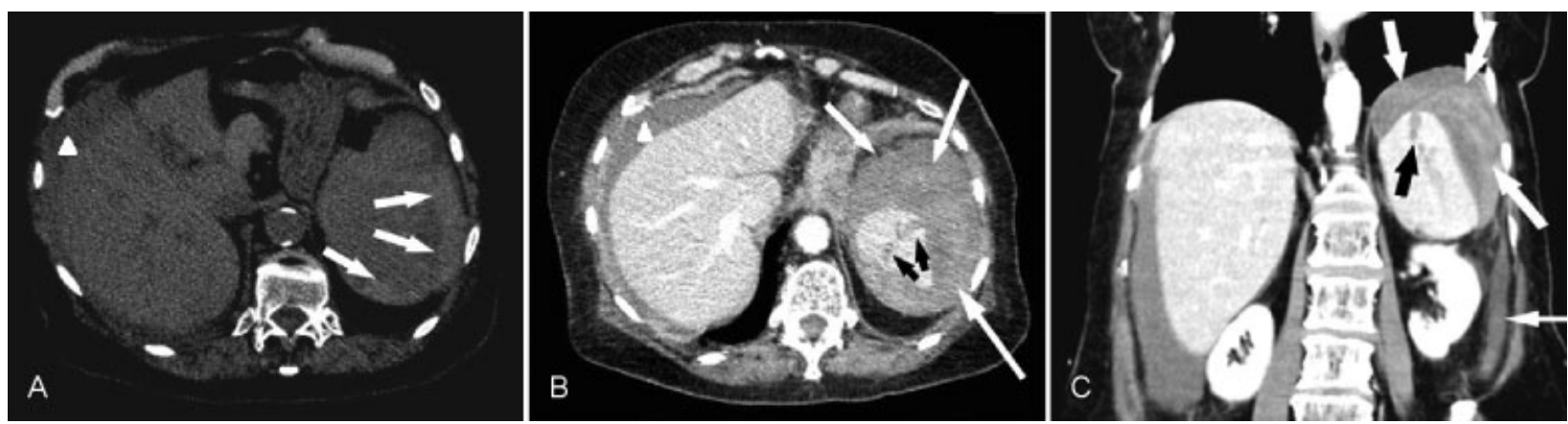

Fig. 7 Paciente de 85 años presentó síncope vasovagal con traumatismo abdominal. En (A), plano axial sin contraste que demostró área con forma de semiluna espontáneamente hiperdensa correspondiente a hematoma subcapsular esplénico (flechas blancas) asociado a líquido perihepático (cabeza de flecha), en fase portal (B) se evidenció laceración esplénica (flechas negras) junto a hematoma subcapsular (entre flechas blancas) y líquido libre perihepático (cabeza de flecha). (C) Reconstrucción coronal en fase portal, laceración esplénica (flecha negra), hematoma subcapsular con progresión temporal (flechas blancas). Aparente ruptura del hematoma subcapsular con pasaje de sangre al espacio parietocólico izquierdo y derecho (flecha fina). 

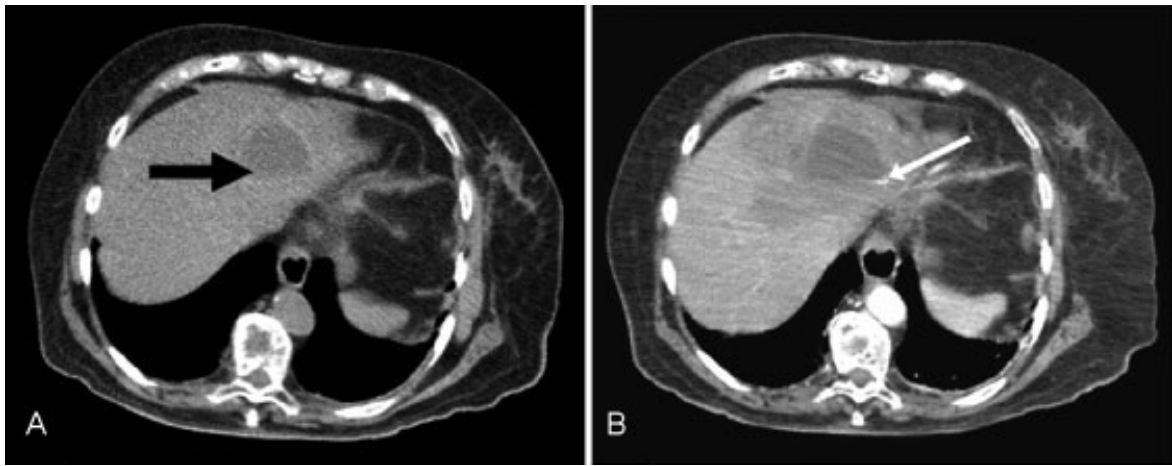

Fig. 8 Paciente de 90 años, presentó caída de propia altura con traumatismo abdominal. En (A), plano axial sin contraste IV que evidenció lesión focal hipodensa que aparenta tener un nivel de contenido espontáneamente hiperdenso correspondiente a hematoma (flecha negra). En fase arterial: (B) falta de realce de la lesión descrita en (A) asociada a imagen hiperdensa correspondiente a extravasación de contraste de forma activa (flecha blanca).

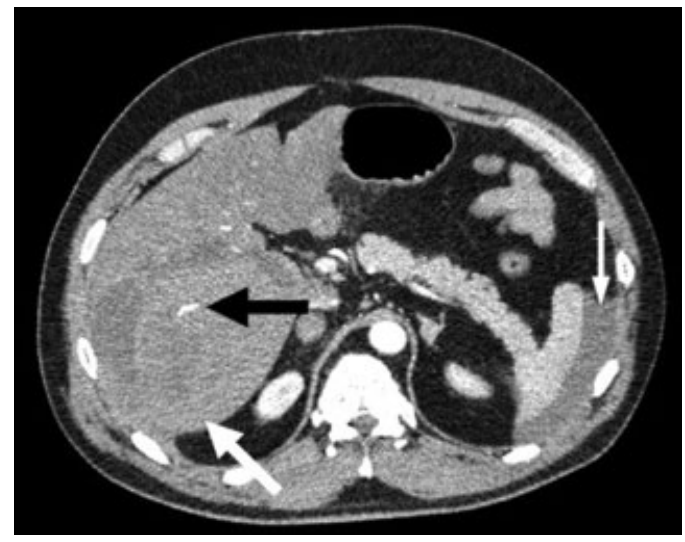

Fig. 9 Paciente de 58 años anticoagulado, presentó politraumatismo por accidente en motocicleta. En (A), plano axial en fase arterial. Se evidenció extensa lesión espontáneamente hiperdensa localizada en los segmentos V, VI y VII hepáticos compatible con un hematoma subcapsular (flecha blanca gruesa) con fuga del material de contraste (flecha negra) y líquido libre periesplénico (flecha fina blanca).

adyacente. Para diferenciarlo de una hemorragia activa, se visualiza en la fase tardía por la falta de lavado del contraste. Los pseudoaneurismas suelen ser embolizados para evitar su ruptura y sangrado posterior ${ }^{19}$ (- Figs. 10 y 11).
F) Hemoperitoneo: El sangrado comienza cercano a la lesión parenquimatosa y sigue su camino anatómico. En las lesiones esplénicas, la sangre extravasada fluye en dirección caudal desde el espacio periesplénico a lo largo del espacio parietocólico izquierdo hacia la pelvis. La hemorragia desde el hígado fluye en dirección caudal desde el espacio perihepático y la fosa hepatorrenal a través del espacio parietocólico derecho hacia el espacio rectovesical en el hombre y recto uterino en la mujer. En ocasiones, un gran volumen sanguíneo puede acumularse en la pelvis sin que hubiera una colección sanguínea alrededor del órgano fuente. $^{20} \mathrm{El}$ análisis de la atenuación en Unidades de Hounsfield (UH), es una herramienta útil para evidenciar sangrado reciente y cercano a la fuente $(45-70 \mathrm{UH})^{1}$ (-Fig. 12).

Las complicaciones tardías en las lesiones esplénicas y hepáticas no son comunes y ocurren dentro de las 48 horas posteriores al trauma e incluyen: pseudoquistes (-Fig. 13), abscesos, pseudoaneurismas, ruptura con hemorragia tardía, hemobilia (extravasación sanguínea visualizada como material hiperdenso dentro de la vía biliar), bilomas (colección de bilis dentro del parénquima hepático visualizado como una hipodensidad homogénea de contornos bien definidos).
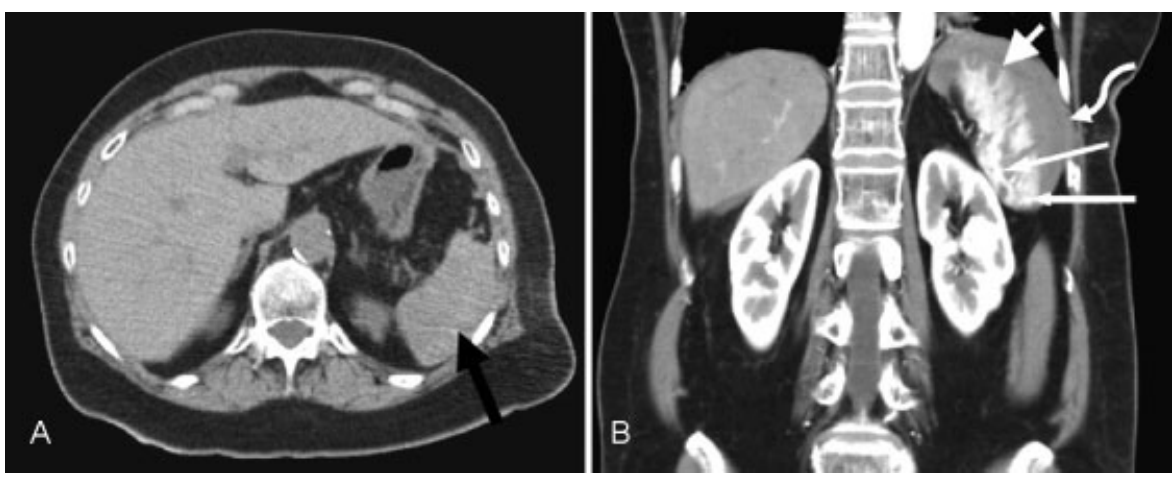

Fig. 10 Paciente de 73 años politraumatizado, secundario a caída de propia altura, presentó descenso del hematocrito, en (A) plano axial sin contraste con tenue área espontáneamente hiperdensa en el parénquima esplénico (flecha). (B) Reconstrucción coronal con contraste IV: múltiples lesiones hipodensas correspondientes a laceraciones esplénicas (flecha corta), hematoma subcapsular esplénico (flecha curva) y pseudoaneurismas (flechas largas) ya que se continúan visualizando en una adquisición tardía, confirmadas por angiografía digital. 

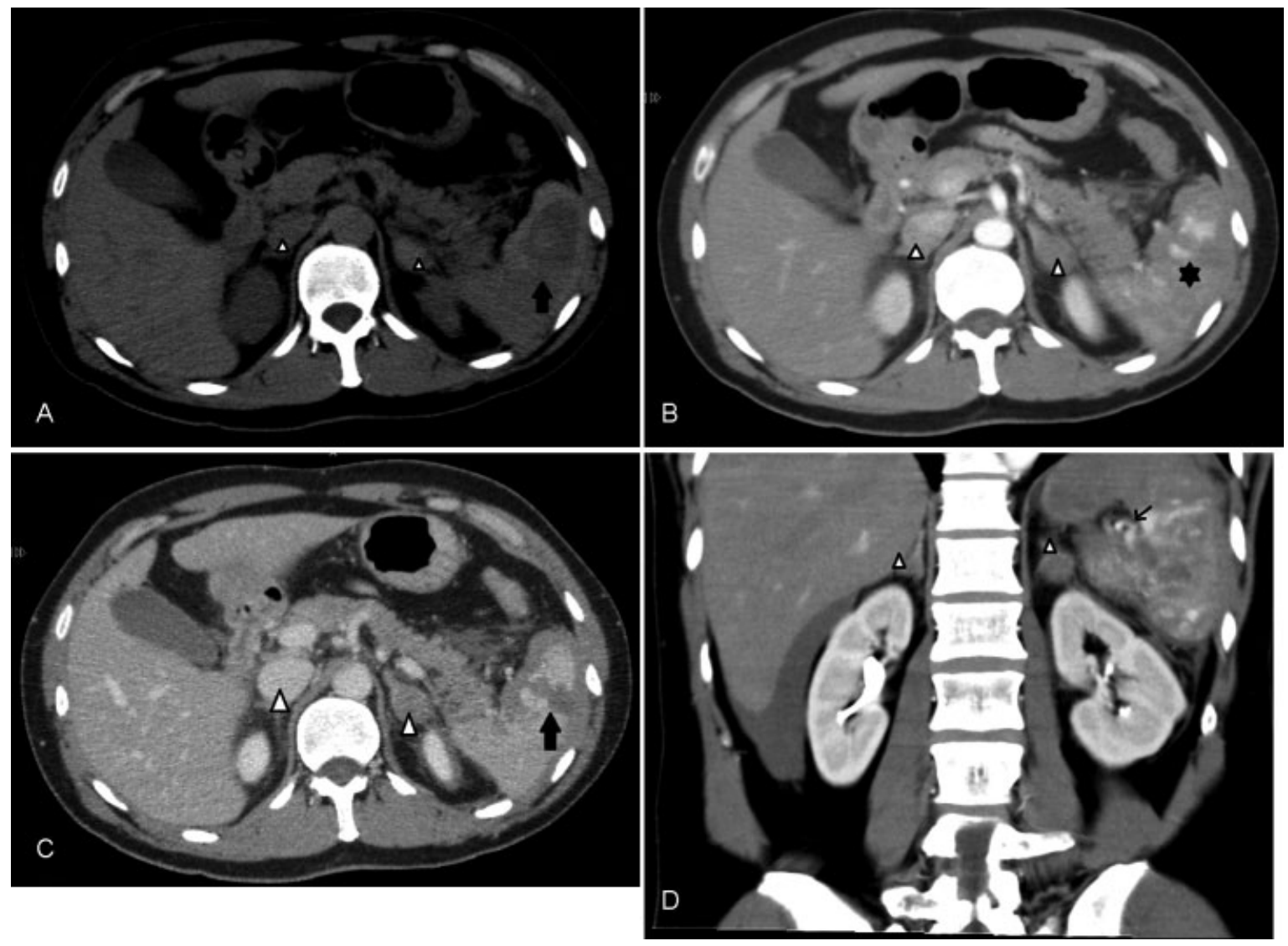

Fig. 11 Paciente de 50 años, ingresó por politraumatismo secundario a accidente en vía pública. Presentó hematomas suprarrenales. (A) Plano axial sin contraste. Extensa área hipodensa en el parénquima esplénico correspondiente a laceración (flecha gruesa) y aumento espontáneo en la densidad de ambas glándulas suprarrenales correspondientes a hematomas suprarrenales (cabeza de flecha). Al administrar contraste IV en fase arterial (B) se evidenció extravasación activa de contraste dentro del parénquima esplénico (estrella) y leve aumento de la densidad de ambos hematomas suprarrenales (cabeza de flecha). En fase portal (C), se evidenció permanencia de contraste extravasado dentro de laceración esplénica (flecha gruesa), y hematomas suprarrenales (cabeza de flecha). (D) Reconstrucción coronal en fase tardía que informó permanencia de contraste dentro de laceración esplénica, pudiendo corresponder a pseudoaneurisma (flecha fina), líquido en espacio parietocólico derecho y hematomas suprarrenales (cabeza de flecha).

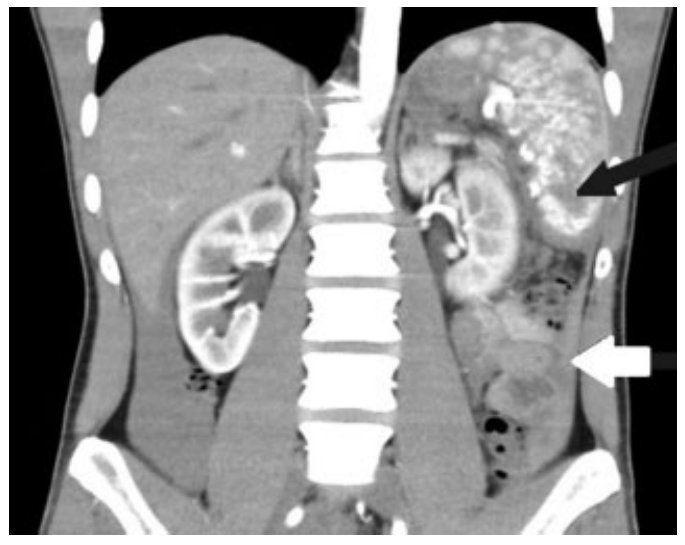

Fig. 12 Paciente con laceración esplénica conocida secundario a caída de propia altura presentó descenso del hematocrito. Reconstrucción coronal de TCMC control con contraste IV en fase arterial, donde se visualizó laceración esplénica (flecha negra) y hemoperitoneo que discurre desde el espacio periesplénico hacia la gotera parietocólico izquierda (flecha blanca).

El hemoperitoneo usualmente se resuelve en una semana, el hematoma subcapsular entre 6 y 7 semanas. Las laceraciones en 3 semanas. Hematomas intraparenquimatosos y bilomas pueden persistir por años. $^{21,22}$ Los cambios normales en la cicatrización de las lesiones no deben ser confundidos con complicaciones. La realización de tomografías de control seriadas no está indicada de forma sistemática. ${ }^{23}$

\section{Conclusión}

La TCMC ha demostrado ser, gracias a su elevada resolución temporo-espacial y su consecuente calidad de imágenes, una herramienta indispensable para la identificación de los variados patrones de lesión de órganos sólidos abdominales y lesiones asociadas que pueden requerir tratamiento urgente, demostrando utilidad también en el seguimiento, monitoreo y detección de complicaciones tardías.

Confidencialidad de los datos

Los autores declaran que han seguido los protocolos de su centro de trabajo sobre la publicación de datos de pacientes, y que todos los pacientes incluidos en el estudio, han recibido información suficiente y han dado su consentimiento informado por escrito. 

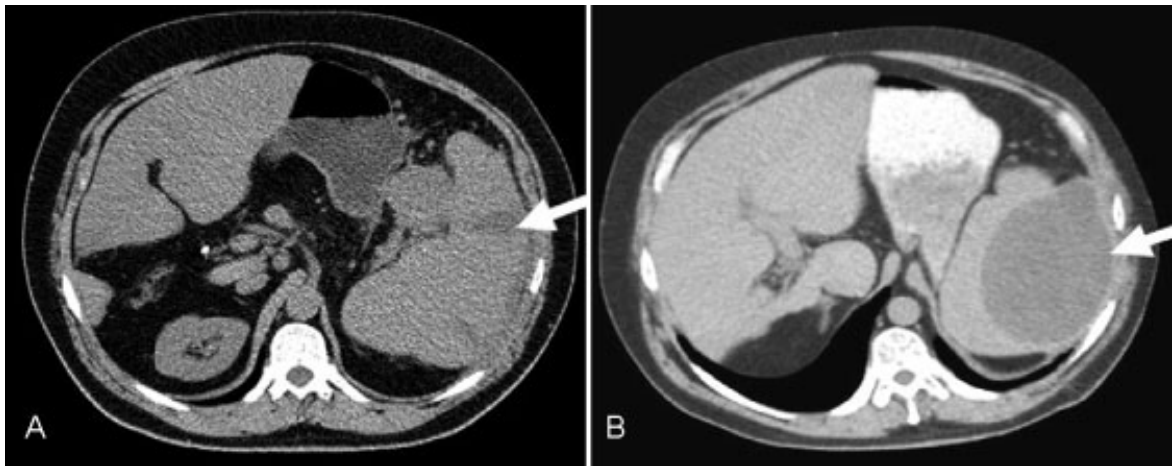

Fig. 13 Paciente de 56 años con antecedentes de traumatismo contuso en hipocondrio izquierdo que presentó hematoma intraparenquimatoso esplénico. TCMC de control presentó lesión pseudoquística, visualizada en (A), plano axial sin contraste con colección levemente hiperdensa correspondiente a hematoma intraparenquimatoso esplénico (flecha). (B) control bimestral. Plano axial con contraste IV. Lesión hipodensa de bordes bien definidos de apariencia quística que desplaza al parénquima circundante (flecha), correspondiente a pseudoquistes.

Conflicto de intereses

Los autores declaran no tener ningún conflicto de intereses.

\section{Bibliografía}

1 Soto JA, Anderson SW. Multidetector CT of blunt abdominal trauma. Radiology 2012;265(03):678-693

2 Dr. Konfino J. Boletín de vigilancia Enfermedades no transmisibles y factores de riesgo. Ministerios de Salud de la Nación Argentina web site. Disponible en: http://www.msal.gob.ar/ent/images/ stories/vigilancia/pdf/2016-2_boletin-de-vigilancia-8.pdf. (accedido abril 2018)

3 Kailidou E, Pikoulis E, Katsiva V, et al. Contrast-enhanced spiral CT evaluation of blunt abdominal trauma. JBR-BTR 2005;88(02): 61-65

4 Yao DC, Jeffrey RB Jr, Mirvis SE, et al. Using contrast-enhanced helical CT to visualize arterial extravasation after blunt abdominal trauma: incidence and organ distribution. AJR Am J Roentgenol 2002;178(01):17-20

5 Matthes G, Stengel D, Seifert J, Rademacher G, Mutze S, Ekkernkamp A. Blunt liver injuries in polytrauma: results from a cohort study with the regular use of whole-body helical computed tomography. World J Surg 2003;27(10):1124-1130

6 Hoff WS, Holevar M, Nagy KK, et al; Eastern Asociation for the Surgery of Trauma. Practice management guidelines for the evaluation of blunt abdominal trauma: the East practice management guidelines work group. J Trauma 2002;53(03): 602-615

7 Hennes HM, Smith DS, Schneider K, Hegenbarth MA, Duma MA, Jona JZ. Elevated liver transaminase levels in children with blunt abdominal trauma: a predictor of liver injury. Pediatrics 1990;86 (01):87-90

8 Becker CD, Mentha G, Terrier F. Blunt abdominal trauma in adults: role of $\mathrm{CT}$ in the diagnosis and management of visceral injuries. Part 1: liver and spleen. Eur Radiol 1998;8(04):553-562

9 Fang JF, Wong YC, Lin BC, Hsu YP, Chen MF. Usefulness of multidetector computed tomography for the initial assessment of blunt abdominal trauma patients. World J Surg 2006;30(02): 176-182

10 Benjamin E, Cho J, Recinos G, et al. Negative computed tomography can safely rule out clinically significant intra- abdominal injury in the asymptomatic patient after blunt trauma: Prospective evaluation of 1193 patients. J Trauma Acute Care Surg 2018;84(01):128-132

11 Schwab CW. Selection of nonoperative management candidates. World J Surg 2001;25(11):1389-1392

12 Neish AS, Taylor GA, Lund DP, Atkinson CC. Effect of CT information on the diagnosis and management of acute abdominal injury in children. Radiology 1998;206(02):327-331

13 Poletti PA, Mirvis SE, Shanmuganathan K, Killeen KL, Coldwell D. CT criteria for management of blunt liver trauma: correlation with angiographic and surgical findings. Radiology 2000;216 (02):418-427

14 Gamanagatti S, Rangarajan K, Kumar A, Jineesh. Blunt abdominal trauma: imaging and intervention. Curr Probl Diagn Radiol 2015; 44(04):321-336

15 Rozycki GS, Ochsner MG, Schmidt JA, et al. A prospective study of surgeon-performed ultrasound as the primary adjuvant modality for injured patient assessment. J Trauma 1995;39(03):492-498, discussion 498-500

16 Doody O, Lyburn D, Geoghegan T, Govender P, Munk PL, Torreggiani WC. Blunt trauma to the spleen: ultrasonographic findings. Clin Radiol 2005;60(09):968-976

17 Blaivas M, Lyon M, Brannam L, Schwartz R, Duggal S. Feasibility of FAST examination performance with ultrasound contrast.J Emerg Med 2005;29(03):307-311

18 van der Vlies CH, van Delden OM, Punt BJ, Ponsen KJ, Reekers JA, Goslings JC. Literature review of the role of ultrasound, computed tomography, and transcatheter arterial embolization for the treatment of traumatic splenic injuries. Cardiovasc Intervent Radiol 2010;33(06):1079-1087

19 Jeffrey RB Jr, Cardoza JD, Olcott EW. Detection of active intraabdominal arterial hemorrhage: value of dynamic contrast-enhanced CT. AJR Am J Roentgenol 1991;156(04): 725-729

20 Lubner M, Menias C, Rucker C, et al. Blood in the belly: CT findings of hemoperitoneum. Radiographics 2007;27(01):109-125

21 Kristoffersen KW, Mooney DP. Long-term outcome of nonoperative pediatric splenic injury management. J Pediatr Surg 2007;42(06):1038-1041, discussion 1041-1042

22 Maull KI. Current status of nonoperative management of liver injuries. World J Surg 2001;25(11):1403-1404

23 Uranüs S, Pfeifer J. Nonoperative treatment of blunt splenic injury. World J Surg 2001;25(11):1405-1407 